\title{
Insulin After 75 Years: Still A Heroic Hormone
}

$\mathrm{T}$ his year marks the 75th anniversary of the discovery of insulin. Banting and Best at the University of Toronto first reported their success in preparing a pancreas extract that dramatically lowered blood glucose levels in diabetic dogs in early $1922 .{ }^{1}$ As early as the Spring of 1922 , remarkably fast time by today's standards, this achievement was followed by successful clinical trials in humans that demonstrated the effectiveness of the pancreas extract in lowering glucose levels and preventing death from diabetic ketoacidosis. Indeed, one of the early patients treated with this extract survived for 59 years and another for more than 70 years: some outcomes for a clinical trial! Banting and Best subsequently won the Nobel Prize for this work.

In medical school we learn about the remarkable powers of this heroic hormone, insulin, which turned out to be the active ingredient in the pancreas extract. Insulin, a lone anabolic hormone, does battle with multiple catabolic hormones such as glucocorticoids, glucagon, and catecholamines to maintain homeostatic balance. In the hospital we observe directly insulin's power to reverse the dramatic metabolic derangements of patients presenting with diabetic ketoacidosis.

\section{See also p 407}

With this as background, as an insulinophile I have been somewhat shocked and disturbed by a number of epidemiological studies suggesting that elevated levels of this wonderful hormone insulin may be detrimental, a marker or independent risk factor for the development of coronary artery disease. ${ }^{2}$ However, increased insulin levels occurring as a physiological response to insulin resistance are associated with a number of other metabolic abnormalities, including hypertension, central body adiposity, and lipid abnormalities. ${ }^{3}$ When these other known risk factors for coronary artery disease are controlled for, evidence for an independent adverse effect of insulin has not been very consistent. ${ }^{4,5}$ Recently, a study of men from Quebec, using a matched pair design to control for other risk factors, again suggested that hyperinsulinemia independently predicts development of coronary artery disease. ${ }^{6}$ Thus, it was with some relief that I read the paper by Lindberg, et al. in the current issue of the Journal of the American Geriatrics Society indicating that in cohorts of older Finnish men, prospective follow-up demonstrated no adverse effect of a higher insulin level on either development of coronary heart disease or survival ${ }^{7}$. In fact, there is a suggestion of better survival in those with higher insulin levels, a bonus finding for an insulinophile like me. Although patients in this study who have cardiovascular disease crosssectionally tend to have higher insulin levels, the authors conclude that this elevation of insulin is secondary to the cardiovascular disease rather a contributing factor.
A number of investigators have developed a rationale for potential adverse effects of increased insulin levels. Insulin has direct cardiovascular effects, ${ }^{8}$ some of which could contribute to the development of hypertension, which in turn causes coronary artery disease. Insulin can also lead to alterations of lipid metabolism ${ }^{9}$ and is reported to have potentially atherogenic effects on vascular smooth muscle in vitro. $^{10}$

After 75 years, where do we stand regarding benefits and potential risks of insulin? Epidemiologists sometimes describe J-shaped curves in predictions of mortality. For example, Andres has demonstrated that although increasing adiposity is associated with increased mortality, there is also an association of diminished adiposity with increasing mortality such that the degree of adiposity for best survival lies between low and high. ${ }^{11}$ Perhaps the relationship between insulin and coronary artery disease or mortality is a reversed J. It is very clear that marked insulin deficiency is a direct cause of death and that insulin-deficient people with diabetes are at substantially increased risk for development of coronary artery disease and other diabetes complications. ${ }^{12}$ Although insulin resistance is present in patients with diabetes mellitus, particularly NIDDM, these patients with overt hyperglycemia have beta cell failure and low rather than high insulin levels. ${ }^{13}$ It should also be noted that a number of previous studies have overestimated circulating insulin levels in people with diabetes because commonly used insulin assays measure both insulin and proinsulin, and there is now evidence that patients with overt diabetes have an increased percentage of circulating proinsulin. ${ }^{13}$ Thus, there is little doubt that low insulin levels are associated with adverse outcomes. There may also be a small increased risk associated with chronic exposure to elevated insulin levels as occurs in people with insulin resistance (but not diabetes). However, this remains controversial and may be explained by a number of other risk factors that are associated with insulin resistance but not caused by it. At least among Finnish men who have survived to the geriatric age cohort, increased circulating insulin levels may be a better marker of survival than of adverse events. Further conclusions about potential risks of hyperinsulinemia in other populations and in women will await additional studies. In the meantime, let's celebrate the 75th anniversary of the discovery of insulin with the enthusiasm that it deserves.

\section{Jeffrey B. Halter, MD \\ University of Michigan Medical School, and Geriatric Research Education and Clinical Center Veterans Affairs Medical Center} Ann Arbor, MI

\section{REFERENCES}

1. Banting FG, Best $\mathrm{CH}$. The internal secretion of the pancreas. J Lab Clin Med $1922 ; 7: 465-480$. 
2. Stout RW. Insulin and atheroma: 20-year perspective. Diabetes Care 1990;13:631-654

3. Foster D. Insulin resistance-A secret killer? N Engl J Med 1989;320:733734.

4. McKeigue P, Davey G. Associations between insulin levels and cardiovascular disease are confounded by comorbidity. Diabetes Care 1995;18:12941298.

5. Wingard DL, Barrett-Connor EL, Ferrara A. Is insulin really a heart disease risk factor? Diabetes Care 1995;18:1299-1304.

6. Despres JP, Lamarche B, Mauriege $P$ et al. Hyperinsulinemia as an independent risk factor for ischemic heart disease. N Engl J Med 1996;334:952-957.

7. Lindberg $\mathrm{O}$, Tilvis RS, Strandberg TE et al. Elevated fasting plasma insulin in a general aged population: An innocent companion of cardiovascular diseases. J Am Geriatr Soc 1997;45:407-412.

8. Baron AD. Hemodynamic actions of insulin. Am J Physiol 1994; 267(Endo- crinol. Metab. 30):E187-202.

9. Ostlund RE Jr, Staten M, Kohrt WM et al. The ratio of waist-to-hip circumference, plasma insulin level, and glucose tolerance as independent predictors of the $\mathrm{HDL}_{2}$ cholesterol level in older adults. N Engl J Med 1990; 322:229234.

10. Stout RW. Insulin as a mitogenic factor: Role in the pathogenesis of cardiovascular disease. Am J Med 1991;90(suppl 2A):62-65S.

11. Andres R: Mortality and obesity: The rationale for age-specific height-weight tables. In: Hazzard WR, Bierman EL, Blass JP, et al., eds. Principles of Geriatric Medicine and Gerontology, 3rd Ed. New York: McGraw-Hill, 1994, p. 847.

12. Carter Center of Emory University. Closing the gap: The problem of diabetes mellitus in the United States. Diabetes Care 1985;8:391-406.

13. Porte D Jr. $\beta$-cells in Type II diabetes mellitus. Diabetes $1991 ; 40: 166-180$, 